\title{
A new method for the numerical evaluation of domain integrals in a 3D boundary element method for transient heat conduction
}

\author{
Y. Dong, J. Zhang, G. Xie, C. Lu, L. Han \& P. Wang \\ State Key Laboratory of Advanced Design and Manufacturing \\ for Vehicle Body, Hunan University, China
}

\begin{abstract}
A new method is proposed for the numerical evaluation of domain integrals in a 3D boundary element method. These integrals arise in the solution of the transient heat conduction problems, using a time-dependent boundary integral equation method named as pseudo-initial condition method. As the time-dependent kernel in the domain integral is close to singular when small time step is used, a straightforward application of Gaussian quadrature may produce large errors, and thus lead to instability of the analysis. In this paper, a coordinate transformation coupled with an element subdivision technique is presented. The coordinate transformation is denoted as $(\alpha, \beta, \gamma)$ transformation, while the element subdivision technique considers the position of the source point, the property of the time-dependent fundamental solution and the relations between the size of the element and the time step. With the coordinate transformation and the element subdivision technique, more Gaussian points are shifted towards the source point, thus more accurate results can be obtained. Numerical examples have demonstrated the accuracy and efficiency of the proposed method.
\end{abstract}

Keywords: time-dependent, domain integrals, transient heat conduction, boundary element method, element subdivision technique.

\section{Introduction}

The transient heat conduction problem widely appears in engineering problem. The boundary element method (BEM) is a very attractive method to analyze this problem [1-10]. The implementation of BEM based on time-dependent 
fundamental solution for transient heat conduction problem can be classified into two kinds: the time convolution method and the pseudo-initial condition method. In the time convolution method, temperature and flux at each step are computed through a convolution of temperature and flux on the boundary at previous steps. If the initial temperature and the heat generation are omitted, the time convolution method leads to a pure boundary method. However, as indicated in [4-8], the time convolution method suffers from the time-consuming convolution especially in the case that a long time history is concerned. Compared with the time convolution method, no time consuming convolution is required in the pseudo-initial condition method. Therefore, the pseudo-initial condition method is more widely used than the time convolution method in engineering problem. When using the pseudoinitial condition method, the temperature computed in the previous step is considered as the initial condition in current step. Thus, the domain integral of this pseudo-initial condition is required in this method.

Although the domain integrals are actually regular in nature, they can't be evaluated accurately and efficiently by the standard Gaussian quadrature when the small time step is used. This is because as the time step approaches zero, the integrand in the domain integral (the time-dependent fundamental solution) is close to singular as the source point is on the integration element. The difficulty of numerically integrating a function with such behavior can introduce numerical unstable problems into the solution, as reported in [11-14]. Thus accurate calculation of the domain integrals is important for the successful implementation of the pseudo-initial condition method.

Various methods within the scope of BEM have been proposed to cope with such problem. Sharp investigated that if the time step is chosen to be too small, the accuracy of the approximation deteriorates. And a stability condition [11] was derived to avoid the problem. Peirce et al investigated that the size of the spatial mesh relative to the time step affected the accumulation of errors in the one-step recursion scheme. Thus they introduced a dimensionless meshing parameter [12] whose magnitude governs the performance of the one-step BEM. The main drawback of the two methods is that if the time step is very small, the computational cost is huge according to requirement of these methods. The authors think that the bottleneck is how to accurately calculate the domain integrals which are involved with the time-dependent fundamental solution. So it is time to develop a new method for evaluating the domain integrals accurately and efficiently. This paper presents a new method for the domain integrals. The new method inherits advantages of the Sharp's stability condition and Peirce's dimensionless meshing parameter. Moreover, a new coordinate transformation coupled with an element subdivision technique is introduced to evaluate the domain integrals. Thus the influence of the size of the spatial mesh relative to the time step is weakened.

In our method, firstly a coordinate transformation denoted as $(\alpha, \beta, \gamma)$ transformation is introduced. It is an extension of Zhang's $(\alpha, \beta)$ transformation [15]. Furthermore, an element subdivision technique is proposed considering the position of the source point, the shape of the element and a dimensionless meshing 
parameter which is proposed by Peirce et al. With the element subdivision technique, integration elements are divided into pyramidal and hexahedral patches. And the rapid variations of the integrand are smoothed out by using the $(\alpha, \beta, \gamma)$ transformation on pyramidal patches. Thus the integrands of domain integrals which vary drastically can be accurately calculated by our method even if the time step is very small. Numerical examples are presented to verify our method. Results demonstrate the accuracy and efficiency of our method.

This paper is organized as follows. In section 2 , the boundary integral equation and the domain integral are described. Section 3 introduces the $(\alpha, \beta, \gamma)$ transformation and the element subdivision technique. Numerical examples are given in Section 4. The paper ends with conclusions in Section 5.

\section{General description}

\subsection{The boundary integral equation}

In this section, we study boundary integral solutions to the diffusion equation

$$
\nabla^{2} u(\mathbf{x}, t)-\frac{1}{k} \frac{\partial u(\mathbf{x}, t)}{\partial t}=0
$$

The boundary integral equation for transient heat conduction in an isotropic, homogeneous medium $\Omega$ bounded by $\Gamma$ is given by:

$$
\begin{aligned}
& c(\mathbf{y}) u\left(\mathbf{y}, t_{F}\right)+k \int_{t_{0}}^{t_{F}} \int_{\Gamma} u(\mathbf{x}, t) q^{*}\left(\mathbf{y}, \mathbf{x}, t_{F}, t\right) d \Gamma(\mathbf{x}) d t \\
& =k \int_{t_{0}}^{t_{F}} \int_{\Gamma} q(\mathbf{x}, t) u^{*}\left(\mathbf{y}, \mathbf{x}, t_{F}, t\right) d \Gamma(\mathbf{x}) d t+\int_{\Omega} u_{0}\left(\mathbf{x}, t_{0}\right) u^{*}\left(\mathbf{y}, \mathbf{x}, t_{F}, t_{0}\right) d \Omega(\mathbf{x})
\end{aligned}
$$

where $\mathbf{y}$ and $\mathbf{x}$ are the source and the field points, respectively. $c(\mathbf{y})$ is a function of the solid angle of the boundary at point $\mathbf{y} . k$ denotes the diffusion coefficient, $t_{0}$ stands for the initial time and $u_{0}\left(\mathbf{x}, t_{0}\right)$ is the initial condition.

The time-dependent fundamental solution $u^{*}$ is as follows:

$$
\begin{gathered}
u^{*}=\frac{1}{(4 \pi k \tau)^{1.5}} \exp \left(-\frac{r^{2}}{4 k \tau}\right) \\
q^{*}=\partial u^{*} / \partial n
\end{gathered}
$$

where $\tau=t_{F}-t, r$ represents the Euclidean distance between the source and the field points, and $n$ is the unit outward normal at the boundary.

\subsection{The domain integral}

The domain integral involved in Eq. (2) is as follows:

$$
I=\int_{\Omega} u_{0}\left(\mathbf{x}, t_{0}\right) u^{*}\left(\mathbf{y}, \mathbf{x}, t_{F}, t_{0}\right) d \Omega(\mathbf{x})=\int_{\Omega} u_{0}\left(\mathbf{x}, t_{0}\right) \frac{1}{4 \pi k \tau} \exp \left(-\frac{r^{2}}{4 k \tau}\right) d \Omega(\mathbf{x})
$$


The initial condition $u_{0}\left(\mathbf{x}, t_{0}\right)$ is a regular function. As the time step approaches zero, the integrand in the domain integral (the time-dependent fundamental solution $u^{*}$ ) is close to singular as shown in Fig. 1. Thus the domain integrals cannot be accurately calculated by the standard Gaussian quadrature when small time step is used.
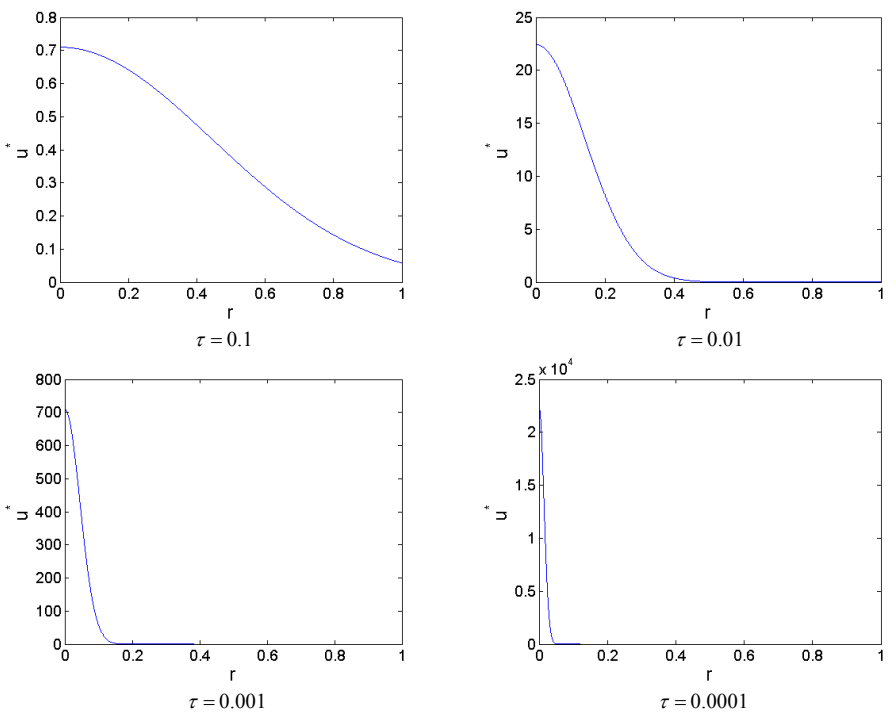

Figure 1: Variation of $u^{*}$ with $r$ for several values of time steps.

\section{New method for evaluating the domain integrals}

\subsection{The $(\alpha, \beta, \gamma)$ transformation}

In this section, we first introduce the $(\alpha, \beta, \gamma)$ transformation. The transformation is used in the following sub-pyramid, and this is a method for solving the singular integrals. To construct the $(\alpha, \beta, \gamma)$ coordinate system as shown in Fig. 2, the following mapping is used:

$$
\left\{\begin{array}{l}
\left\{\begin{array}{l}
x_{\gamma}=x_{1}+\left(x_{2}-x_{1}\right) \alpha+\left(x_{4}-x_{1}\right) \beta \\
y_{\gamma}=y_{1}+\left(y_{2}-y_{1}\right) \alpha+\left(y_{4}-y_{1}\right) \beta \quad \alpha, \beta \in[0,1] \\
z_{\gamma}=z_{1}+\left(z_{2}-z_{1}\right) \alpha+\left(z_{4}-z_{1}\right) \beta
\end{array}\right. \\
\left\{\begin{array}{l}
x=x_{0}+\left(x_{\gamma}-x_{0}\right) \gamma \\
y=y_{0}+\left(y_{\gamma}-y_{0}\right) \gamma \quad \gamma \in[0,1] \\
z=z_{0}+\left(z_{\gamma}-z_{0}\right) \gamma
\end{array}\right.
\end{array}\right.
$$


Combining Eqs. (6a)-(6b), the expression for obtaining coordinates $(x, y, z)$ can be written as:

$$
\left\{\begin{array}{l}
x=x_{0}+\left[\left(x_{1}-x_{0}\right)+\left(x_{2}-x_{1}\right) \alpha+\left(x_{4}-x_{1}\right) \beta\right] \gamma \\
y=y_{0}+\left[\left(y_{1}-y_{0}\right)+\left(y_{2}-y_{1}\right) \alpha+\left(y_{4}-y_{1}\right) \beta\right] \gamma \\
z=z_{0}+\left[\left(z_{1}-z_{0}\right)+\left(z_{2}-z_{1}\right) \alpha+\left(z_{4}-z_{1}\right) \beta\right] \gamma
\end{array}\right.
$$

The Jacobian of the transformation from the $(x, y, z)$ system to the $(\alpha, \beta, \gamma)$ system is

$$
J b=\gamma^{2}\left|\begin{array}{ccc}
x_{2}-x_{1} & y_{2}-y_{1} & z_{2}-z_{1} \\
x_{3}-x_{1} & y_{3}-y_{1} & z_{3}-z_{1} \\
x_{1}-x_{0} & y_{1}-y_{0} & z_{1}-z_{0}
\end{array}\right|
$$

With the $(\alpha, \beta, \gamma)$ transformation, the rapid variations of the integrand are smoothed out in some degree. Thus, the computational accuracy of the domain integrals can be improved.

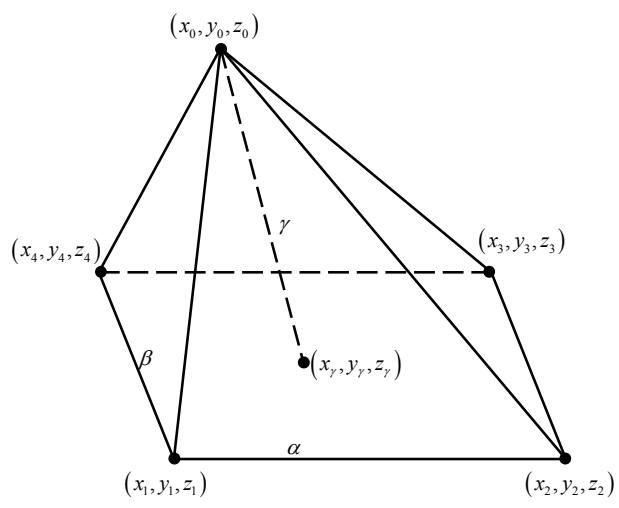

Figure 2: The $(\alpha, \beta, \gamma)$ coordinate transformation.

\subsection{Element subdivision}

To further improve the computational accuracy of the domain integrals, an element subdivision technique is proposed in this part. From the Fig. 1, it can be seen that a large spike occurs in the integrand near the source point as the time step value is small. Thus the steep slopes produced by the integrand require that integration points be shifted towards the source point in order to evaluate more accurately the integral under consideration. The detailed analysis is as follows.

Firstly we study the probability density function of normal distribution

$$
f(x)=\frac{1}{\sqrt{2 \pi} \sigma} \exp \left(-\frac{x^{2}}{2 \sigma^{2}}\right)
$$




$$
\int_{-\infty}^{\infty} \frac{1}{\sqrt{2 \pi} \sigma} \exp \left(-\frac{x^{2}}{2 \sigma^{2}}\right) d x=1
$$

From Eq. (9), it can be noted that the form of the probability density function is almost like that of the time-dependent fundamental solution $u^{*}$. As all we know, the integral value of Eq. (10) mainly concentrates in the interval $[-3 \sigma, 3 \sigma]$. This also applies similarly to the evaluation of the domain integral for the timedependent fundamental solution. Then a length parameter $\lambda \sqrt{k \tau}$ is introduced, and $\sqrt{k \tau}$ is similar to $\sigma$ in the probability density function.

Through the above analysis, the following element subdivision technique is introduced as shown in Fig. 3:

- Firstly, a cube region with the length of $2 \lambda \sqrt{k \tau}$ is constructed to well cover the source point on the integration element. If the cube region beyond the boundary of the element, taking that of the element as the boundary of the cube region.

- Secondly, sub-pyramids are created in cube region considering the position of the source point and sub-hexahedrons are constructed in the element's remaining regions.

The advantage of the proposed element subdivision technique is that more integration points are shifted towards the source point. Using the element subdivision technique coupled with the $(\alpha, \beta, \gamma)$ transformation, the domain integrals can be accurately calculated.

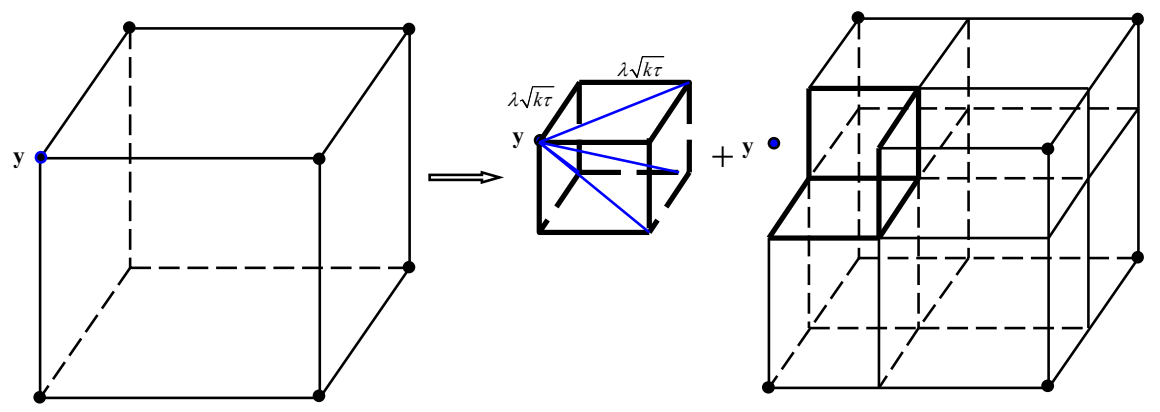

Figure 3: The subdivision of hexahedron element.

\section{Numerical examples}

To verify the accuracy and efficiency of our method, several examples are presented in this section. The domain integrals of the following form are considered:

$$
I=\int_{\Omega} \frac{1}{(4 \pi k \tau)^{1.5}} \exp \left(-\frac{r^{2}}{4 k \tau}\right) d \Omega
$$


The coefficient $k$ in Eq. (11) is assumed to be 1 and the dimensionless parameter $\lambda$ is 8 . The $(\alpha, \beta, \gamma)$ transformation with $10 \times 10 \times 15$ Gaussian points is used on the sub-pyramids and $5 \times 5 \times 5$ point Gaussian quadrature is used on the sub-hexahedrons.

The numerical values obtained by our method will be compared to 'exact' values in terms of the relative error defined by

$$
\text { Relative Error }=\left|\frac{I_{\text {numerical }}-I_{\text {exact }}}{I_{\text {exact }}}\right|
$$

where $I_{\text {numerical }}$ and $I_{\text {exact }}$ are the numerical and 'exact' values of the integral under consideration, respectively. The accuracy of $I_{\text {exact }}$ is to 12 decimal places.

\subsection{Example 1}

In the first example, the domain integral of Eq. (11) is evaluated over a hexahedron element with the node coordinates of $(-1,-1,-1),(-1,1,-1),(-1,1,1),(-1,-1,1)$, $(1,-1,-1),(1,1,-1),(1,1,1),(1,-1,1)$ as shown in Fig. 4 . The coordinate of the source point is set at $(1,1,1)$. The relative errors of various methods with different time steps are compared in Table 1. $\tau$ represents the time step value. $5 \times 5 \times 5$ means straightforward Gaussian quadrature with $5 \times 5 \times 5$ Gaussian points and the $(\alpha, \beta, \gamma)$ transformation combined with the element subdivision technique is denoted as $(\alpha, \beta, \gamma)$.

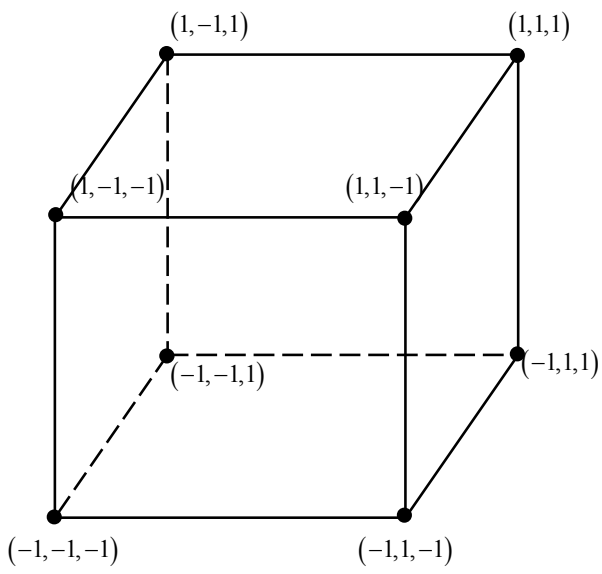

Figure 4: The node coordinates of hexahedron element.

A number of interesting points can be drawn from Table 1:

1. As the time step is large, accurate numerical results can be obtained by applying Gaussian quadrature straightforward, and better accuracy can be obtained with more Gaussian points. 
2. The standard Gaussian quadrature becomes inefficient and inaccurate to evaluate the domain integral when the time step is smaller than 0.001 .

3. Using the proposed method, the domain integral can be accurately and efficiently calculated within a wide range of the time step $\tau$.

As is illustrated in this example, when the time step is very small, the domain integrals cannot be accurately calculated by the standard Gaussian quadrature. However, with our method, high computational accuracy can be obtained within a wide range of the time step $\tau$.

Table 1: Relative errors for integral $I$ on hexahedron element with the node coordinates of $(-1,-1,-1),(-1,1,-1),(-1,1,1),(-1,-1,1),(1,-1,-1)$, $(1,1,-1),(1,1,1),(1,-1,1)$. Errors less than $1 \times 10^{-12}$ are indicated with a '-'.

\begin{tabular}{cccccc}
\hline$\tau$ & 0.1 & 0.01 & 0.001 & 0.0001 & 0.00001 \\
\hline $5 \times 5 \times 5$ & $1.61 \mathrm{E}-03$ & $2.80 \mathrm{E}-01$ & $8.97 \mathrm{E}-01$ & $1.00 \mathrm{E}+00$ & $1.00 \mathrm{E}+00$ \\
$10 \times 10 \times 10$ & $4.31 \mathrm{E}-09$ & $5.73 \mathrm{E}-04$ & $9.73 \mathrm{E}-02$ & $6.78 \mathrm{E}-01$ & $1.00 \mathrm{E}+00$ \\
$15 \times 15 \times 15$ & - & $4.16 \mathrm{E}-06$ & $1.42 \mathrm{E}-02$ & $7.72 \mathrm{E}-01$ & $9.97 \mathrm{E}-01$ \\
$20 \times 20 \times 20$ & - & $4.44 \mathrm{E}-09$ & $1.48 \mathrm{E}-03$ & $8.06 \mathrm{E}-02$ & $1.01 \mathrm{E}-01$ \\
$(\alpha, \beta, \gamma)$ & $1.74 \mathrm{E}-11$ & $1.82 \mathrm{E}-08$ & $4.45 \mathrm{E}-08$ & $4.45 \mathrm{E}-08$ & $4.45 \mathrm{E}-08$ \\
\hline
\end{tabular}

\subsection{Example 2}

In this example, different locations of the source points on the above hexahedron element are considered. $(\alpha, \beta, \gamma)$ represents $(\alpha, \beta, \gamma)$ transformation combined with the element subdivision technique. The relative errors with different time steps are compared in Table 2.

Table 2 shows how good results can be obtained by the method based on $(\alpha, \beta, \gamma)$ transformation considering the different positions of the source points. And the proposed method is not sensitive to the position of the source point.

\subsection{Example 3}

To further demonstrate the effectiveness of the proposed method, a more general example is considered. A cube is heated on the top face and other faces are insulated as shown in Fig.5. The density, heat conductivity and heat capacity are $20 \mathrm{~kg} / \mathrm{m}^{3}, 2 \mathrm{~kJ} /\left(\mathrm{m} .{ }^{\circ} \mathrm{C}\right)$ and $0.8 \mathrm{~kJ} /\left(\mathrm{kg} .{ }^{\circ} \mathrm{C}\right)$, respectively. The length of the 
Table 2: $\quad$ Relative errors for integral $I$ with different position of the source points on hexahedron element with the node coordinates of $(-1,-1$, $-1),(-1,1,-1),(-1,1,1),(-1,-1,1),(1,-1,-1),(1,1,-1),(1,1,1)$, $(1,-1,1)$.

\begin{tabular}{cccccc}
\hline$\tau$ & 0.1 & 0.01 & 0.001 & 0.0001 & 0.00001 \\
\hline$(1.0,0.0,0.0)$ & $1.81 \mathrm{E}-11$ & $6.10 \mathrm{E}-08$ & $1.12 \mathrm{E}-07$ & $1.16 \mathrm{E}-07$ & $1.16 \mathrm{E}-07$ \\
$(1.0,0.0,1.0)$ & $2.65 \mathrm{E}-11$ & $4.20 \mathrm{E}-08$ & $7.88 \mathrm{E}-08$ & $8.04 \mathrm{E}-08$ & $8.04 \mathrm{E}-08$ \\
$(0.9,0.9,0.9)$ & $2.16 \mathrm{E}-11$ & $3.55 \mathrm{E}-08$ & $8.08 \mathrm{E}-07$ & $1.13 \mathrm{E}-07$ & $1.48 \mathrm{E}-07$ \\
$(0.0,0.0,0.0)$ & $1.27 \mathrm{E}-10$ & $7.33 \mathrm{E}-08$ & $1.44 \mathrm{E}-07$ & $1.52 \mathrm{E}-07$ & $1.52 \mathrm{E}-07$ \\
\hline
\end{tabular}

cube is $1 \mathrm{~m}$. A uniform temperature $100^{\circ} \mathrm{C}$ is imposed suddenly on the top face of the cube. The initial temperature of the cube is $0^{\circ} \mathrm{C}$ and the time step is $0.1 \mathrm{~h}$. In this application, the variation history of the temperature from $0 \mathrm{~h}$ to $9.6 \mathrm{~h}$ at the bottom face is concerned. To illustrate the accuracy of the method, numerical results are compared with the existing analytical solution to the considered problem as shown in Fig. 6. Direct solution means using the Gaussian quadrature straightforward for evaluating the domain integrals, and new method represents $(\alpha, \beta, \gamma)$ transformation combined with the element subdivision technique for evaluating the domain integrals.

In Fig. 6, it can be seen that evaluating the domain integrals would cause instability when the time step is $0.1 \mathrm{~h}$ while good results can be obtained with our method.

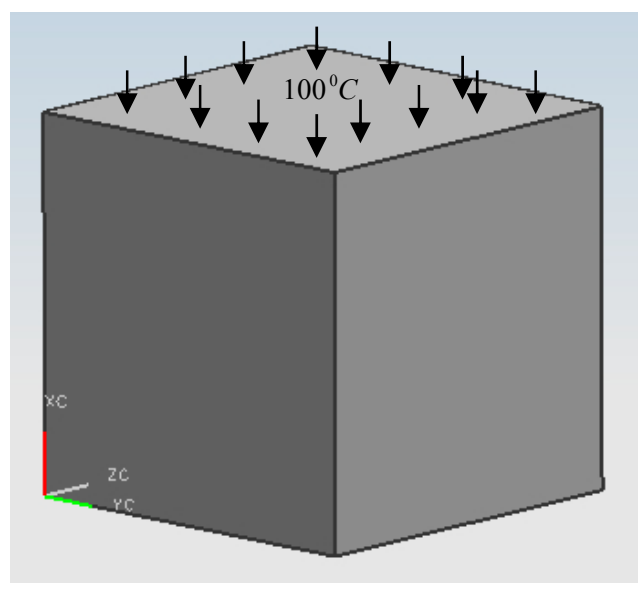

Figure 5: The cube is heated on the top face. 


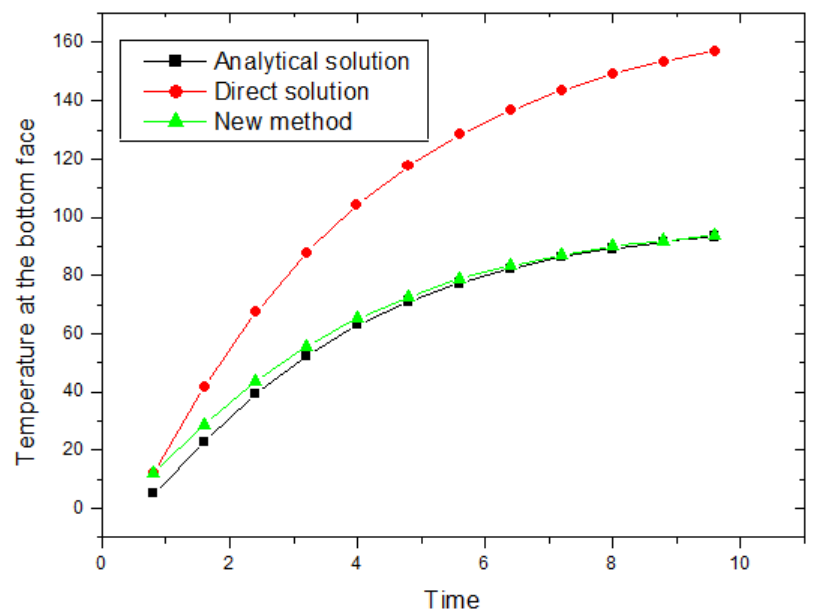

Figure 6: The temperature at the bottom face.

\section{Conclusions}

A general algorithm for the evaluation of the domain integrals which appear in a 3D boundary element method for transient heat conduction problems was proposed in this paper. Employing the proposed method, the influence of the size of the spatial mesh relative to the time step was weakened and the domain integrals can be effectively and accurately calculated. Furthermore, an element subdivision technique takes into account the position of the source point, the shape of the integration element and the relations between the size of element and the time step. Thus even the time step is very small, accurate results can still be obtained by our method. Numerical examples were presented to verify our method. Results demonstrated the accuracy and efficiency of our method.

\section{Acknowledgements}

This work was supported in part by National Science Foundation of China under grant number 11172098, in part by Hunan Provincial Natural Science Foundation for Creative Research Groups of China under grant number 12JJ7001 and in part by Open Research Fund of Key Laboratory of High Performance Complex Manufacturing, Central South University under grant number Kfkt2013-05.

\section{References}

[1] Brebbia, Carlos Alberto, José Claudio Faria Telles, and Luiz C. Wrobel. Boundary element techniques: theory and applications in engineering. Vol. 5. Berlin: Springer-Verlag, 1984.

[2] Sutradhar, Alok, Glaucio H. Paulino, and L. J. Gray. "Transient heat conduction in homogeneous and non-homogeneous materials by the Laplace transform Galerkin boundary element method." Engineering Analysis with Boundary Elements 26.2 (2002): 119-132. 
[3] Hill, L. R., and T. N. Farris. "Fast Fourier transform of spectral boundary elements for transient heat conduction." International Journal of Numerical Methods for Heat \& Fluid Flow 5.9 (1995): 813-827.

[4] Ibáñez, Maria T., and H. Power. "An efficient direct BEM numerical scheme for heat transfer problems using Fourier series." International Journal of Numerical Methods for Heat \& Fluid Flow 10.7 (2000): 687-720.

[5] Gupta, Anil, John M. Sullivan Jr, and Hugo E. Delgado. "An efficient bem solution for three-dimensional transient heat conduction." International Journal of Numerical Methods for Heat \& Fluid Flow 5.4 (1995): 327-340.

[6] Ma, F., et al. "Transient heat conduction analysis of 3D solids with fiber inclusions using the boundary element method." International journal for numerical methods in engineering 73.8 (2008): 1113-1136.

[7] Wang, C. H., M. M. Grigoriev, and G. F. Dargush. "A fast multi-level convolution boundary element method for transient diffusion problems." International Journal for Numerical Methods in Engineering 62.14 (2005): 1895-1926.

[8] Zhou, Fenglin, et al. "Transient heat conduction analysis of solids with small open-ended tubular cavities by boundary face method." Engineering Analysis with Boundary Elements 37.3 (2013): 542-550.

[9] Dargush, G. F., and P. K. Banerjee. "Application of the boundary element method to transient heat conduction." International Journal for Numerical Methods in Engineering 31.6 (1991): 1231-1247.

[10] Guo, Shuaiping, et al. "Three-dimensional transient heat conduction analysis by Laplace transformation and multiple reciprocity boundary face method." Engineering Analysis with Boundary Elements 37.1 (2013): 1522.

[11] Sharp, S. "Stability analysis for boundary element methods for the diffusion equation." Applied Mathematical Modelling 10.1 (1986): 41-48.

[12] Peirce, Anthony P., Attila Askar, and Herschel Rabitz. "Convergence properties of a class of boundary element approximations to linear diffusion problems with localized nonlinear reactions." Numerical Methods for Partial Differential Equations 6.1 (1990): 75-108.

[13] Chang, Y. P., C. S. Kang, and David J. Chen. "The use of fundamental Green's functions for the solution of problems of heat conduction in anisotropic media." International Journal of Heat and Mass Transfer 16.10 (1973): 1905-1918.

[14] Dargush, G. F., and M. M. Grigoriev. "Higher-order boundary element methods for transient diffusion problems. Part II: Singular flux formulation." International Journal for Numerical Methods in Engineering 55.1 (2002): 41-54.

[15] Zhang, Jianming, et al. "A boundary face method for potential problems in three dimensions." International Journal for Numerical Methods in Engineering 80.3 (2009): 320-337. 